\title{
Proceedings of the 26th European Paediatric Rheumatology Congress (PReS 2020) - Late Breaking Abstracts
}

Virtual. 23 - 25 September 2020

Published: 10 December 2020

\section{LB001}

Pediatric Systemic Multi-Inflammatory Diseases in Italy During Sars-Cov-2 Epidemic: From Kawasaki Disease To Kawacovid M. Cattalini', S. Della Paolera ${ }^{2}$, F. Zunica', C. Bracaglia ${ }^{3}$, M. Giangreco ${ }^{4}$, L. Verdoni ${ }^{5}$, A. Meini ${ }^{6}$, R. Sottile, , R. Caorsi ${ }^{8}$, G. Zuccotti ${ }^{9}$, M. Fabi ${ }^{10}$, D. Montin ${ }^{11}$, A. Meneghel $^{12}$, A. Consolaro ${ }^{13}$, R. M. Delle Piane ${ }^{14}$, M. C. Maggio ${ }^{15}$, F. La Torre ${ }^{16}$, A. Marchesi ${ }^{17}$, G. Simonini ${ }^{18}$, A. Villani ${ }^{17}$, R. Cimaz ${ }^{19}$, A. Ravelli $i^{13}$, A. Taddio $^{4}$ ${ }^{1}$ PEDIATRICS CLINIC, UNIVERSITY OF BRESCIA, Brescia; ${ }^{2}$ University of Trieste, Trieste; ${ }^{3}$ Bambino Gesù Children's Hospital, Rome; ${ }^{4}$ IRCCS "Burlo Garofolo", Trieste; ${ }^{5}$ Hospital Papa Giovanni XXIII, Bregamo; ${ }^{6}$ Pediatrics Clinic University of Brescia, Brescia; ${ }^{7}$ Santobono-Pausilipon Children's Hospital, Naples; ${ }^{8}$ IRCCS Istituto Giannina Gaslini, Genova; ${ }^{9}$ Children's Hospital V Buzzi, Milan; ${ }^{10}$ IRCCS S. Orsola-Malpighi, Bologna;

${ }^{11}$ Department of Pediatrics and Public Health, University of Turin, Turin; ${ }^{12}$ University of Padova, Padua; ${ }^{13}$ IRCCS Istituto Giannina Gaslini, Genoa; ${ }^{14}$ Fondazione IRCCS Ca' Granda Ospedale Maggiore Policlinico, Milan; ${ }^{15}$ University of Palermo, Palermo; ${ }^{16 " G i o v a n n i ~ X X I I I ", ~ P e d i a t r i c ~ H o s p i t a l, ~ B a r i, ~}$ Bari; ${ }^{17}$ Bambino Gesu' Children's Hospital, Rome; ${ }^{18} \mathrm{AOU}$ Meyer, University of Florence, Florence; ${ }^{19}$ Department of Clinical Sciences and Community Health, University of Milan, Milan, Italy

Correspondence: $M$. Cattalini

Pediatric Rheumatology 2020, 18(Suppl 3):LB001

Introduction: Italy was affected by the SARS-CoV-2 epidemic after its outbreak in China. With a 4-weeks delay after the peak in adults, we observed an abnormal number of patients with characteristics of a multi-inflammatory disease and similarities with Kawasaki Disease (KD). Others reported similar cases, defined PIMS-TS or MIS-C. ${ }^{1,2}$

Objectives: To better characterize clinical features and treatment response of PIMS-TS and to explore its relationship with KD.

Methods: We conducted an observational, retrospective, multicenter study. On April $24^{\text {th }}-2020$ the Rheumatology Study Group of the Italian Pediatric Society launched a national online survey, to enroll patients diagnosed with KD or with a multisystem inflammatory disease between February $1^{\text {st }} 2020$ and May $31^{\text {st }}$. The population was then divided into two different groups: 1) Classical and incomplete KD, named Kawasaki Disease Group (KDG); 2) KD-like multi-inflammatory syndrome, named KawaCOVID (KCG). An expert panel of pediatric rheumatologists re-analyzed every single patient to ensure appropriate classification. Data were collected with an online database. Results: 149 cases were studied, 96 with KDG and 53 with KCG. The two population significantly differed for clinical characteristics (see table 1). Lymphopenia, higher CRP levels, elevated Ferritin and Troponin-T characterized KCG such as lower WBC and platelets (all $p$ values $<0,05$ ). KDG received more frequently immunoglobulins (IVIG) and acetylsalicylic acid (ASA) (81,3\% vs $66 \% ; p=0.04$ and $71,9 \%$ vs $43,4 \% ; p=0.001$ respectively) as KCG more often received glucocorticoids $(56,6 \%$ vs $14,6 \% ; p<0.0001)$. SARS-CoV- 2 assay more often resulted positive in KCG than in KDG $(75,5 \%$ vs $20 \% ; p<0.0001)$. Short-term follow data on KCG showed minor complications while on KDG a majority of patients had persistence of CAA. Comparing KDG with a KD-Historical Italian cohort (598 patients), no statistical difference was found in terms of clinical manifestations and laboratory data between the two groups

Conclusion: Our study would suggest that SARS-CoV-2 infection might determine two distinct inflammatory diseases in children: KD, possibly triggered by SARS-CoV-2, and PIMS-TS. Older age at onset and clinical peculiarities, like the occurrence of myocarditis, characterize this multiinflammatory syndrome. Our patients had an optimal response to treatments and a good outcome, with few complications and no deaths.

Consent

I have obtained written consent

Disclosure of Interest

None declared

Table 1 (abstract LB001). See text for description

\begin{tabular}{llll}
\hline & KCG & KDG & $\boldsymbol{p}$ value \\
\hline Age at onset & $7(\mathrm{y})$ & $2(\mathrm{y})$ & $<0,0001$ \\
Maculo-papular rash & $61,50 \%$ & $39,60 \%$ & 0,01 \\
Diarrhea & $52,80 \%$ & $11,50 \%$ & $<0,0001$ \\
Tachypnea & $22,60 \%$ & $4,20 \%$ & 0,001 \\
Myocarditis & $60,40 \%$ & $3,10 \%$ & $<0,0001$ \\
ICU admission & $23,10 \%$ & $1,10 \%$ & $<0,0001$ \\
HLH & $18,40 \%$ & $1,20 \%$ & 0,001 \\
Lenght hospitalization & 12 days & 10 days & 0,02 \\
SARS-CoV-2 assay positive & $75,50 \%$ & $20 \%$ & $<0,0001$ \\
\hline
\end{tabular}

(0) The Author(s). 2020 Open Access This article is licensed under a Creative Commons Attribution 4.0 International License, which permits use, sharing, adaptation, distribution and reproduction in any medium or format, as long as you give appropriate credit to the original author(s) and the source, provide a link to the Creative Commons licence, and indicate if changes were made. The images or other third party material in this article are included in the article's Creative Commons licence, unless indicated otherwise in a credit line to the material. If material is not included in the article's Creative Commons licence and your intended use is not permitted by statutory regulation or exceeds the permitted use, you will need to obtain permission directly from the copyright holder. To view a copy of this licence, visit http://creativecommons.org/licenses/by/4.0/ The Creative Commons Public Domain Dedication waiver (http://creativecommons.org/publicdomain/zero/1.0/) applies to the data made available in this article, unless otherwise stated in a credit line to the data. 


\section{LB002}

Immunopathogenesis of Covid-19-Related Pediatric Inflammatory Multisystem Syndrome: Cytokine Profile and Sars-Cov-2 Specific Immune Complexes

A. Esteve-Sole', J. Anton ${ }^{2}$, R. M. Pino-Ramirez ${ }^{3}$, J. Sanchez-Manubens ${ }^{2,4}$, V. Fumadó $^{5}$, C. Fortuny-Guasch ${ }^{6}$, M. Ríos ${ }^{3}$, J. Sanchez de Toledo ${ }^{7}$, M. GironaAlarcón ${ }^{8}$, J. M. Mosquera ${ }^{2}$, S. Ricart ${ }^{3}$, C. Launes ${ }^{3}$, M. Fernández de Sevilla ${ }^{3}$, C. Jou $^{9}$, C. Muñoz-Almagro ${ }^{10}$, E. González-Roca ${ }^{11}$, A. Vergara ${ }^{11,12}$, M. Juan ${ }^{13}$, A. Noguera-Julian ${ }^{5}$, Y. Jordan' ${ }^{8}$, L. Alsina ${ }^{7}$

${ }^{1}$ Allergy and Clinical Immunology; ${ }^{2}$ Pediatric Rheumatology; ${ }^{3}$ Pediatrics, HSJD, BCN; ${ }^{4}$ Pediatric Rheumatology, H. Parc Tauli, Sabadell; ${ }^{5}$ nfectious Diseases, Pediatrics, HSJD; ${ }^{6}$ Infectious Diseases, Pediatrics, SJD; ${ }^{7}$ Cardiology; ${ }^{8}$ Pediatric Intensive Care; ${ }^{9}$ Pathology; ${ }^{10}$ Molecular Microbiology, HSJD; ${ }^{11}$ Molecular Biology Core; ${ }^{12}$ Microbiology Department; ${ }^{13}$ Immunology, CDB, HCB, BCN, Spain Correspondence: L. Alsina

Pediatric Rheumatology 2020, 18(Suppl 3):LB002

Introduction: A multisystem inflammatory syndrome (MIS-C) with temporary association with SARS-CoV-2 pandemics has been recently described in children. MIS-C shares some common features with Kawasaki disease (KD). We hypothesized that: 1) the immune cytokine profiles observed in MIS-C and pre-pandemic KD are different and might explain the different clinical patterns; 2) SARS-CoV-2 specific immune-complexes (IC) may explain the immunopathology of MIS-C

Objectives: To compare the cytokine profile between MIS-C patients, children with SARS-CoV-2 infection without MIS-C, KD patients, and healthy controls $(\mathrm{HC})$; and to explore the presence of circulating SARSCoV-2 specific IC in MIS-C patients.

Methods: Patient's blood samples were drawn within day 1 to 8 of disease onset (prior to any treatment) for the quantification of 34 circulating cytokines (Cytokine \& Chemokine 34-Plex Human ProcartaPlex, ThermoFisher) and evaluation of the presence of circulating SARS-CoV2 IC (TaqPath ${ }^{\text {TM }}$ COVID-19 CE-IVD RT-PCR Kit", ThermoFisher). Protocol was approved by the local ethics committee.

Results: Fifty-eight patients were included: 1 ) 14 with MIS-C (8 positive for SARS-CoV-2 by PCR or serology, mean age 4.58 yo, 50\% male); 2) 10 with positive PCR to SARS-CoV-2 without MIS-C (COVID; mean age 9.5, $50 \%$ male); 3) 14 with pre-pandemic KD (KD; mean age 2.6yo, 57\% male) and 4) 20 pediatric healthy controls (HC; mean age $5.6 y o, 94 \%$ male with negative SARS-CoV-2 $\operatorname{lgG} / \operatorname{lgM} / \lg A$ ).

Compared to HC, MIS-C and KD groups displayed significant higher levels of most cytokines, ranging from 1-6 $\log _{2} \mathrm{FC}$ ( $\log _{2}$ Fold Change). Of these, IFN- $\gamma$-related (IL-18, IFN- $\gamma$, IP-10) and inflammatory monocytes-related cytokines (MCP-1, MIP1- $\beta$, IL-1a, IL-1RA), were the main triggers of inflammation (based on PCA analysis using ClustVis tool). No significant differences were found between MIS-C and KD profiles; however, in a subgroup of patients with MIS-C $\log _{2} \mathrm{FC}$ was greater compared to KD for IFN- $\gamma$, IL-1RA and MCP-1, without reaching statistical significance. A gradation of inflammation is observed between COVID patients, with/without MIS-C.

There was no detection of circulating SARS-CoV-2 IC in MIS-C patients (with or without SARS-CoV-2 confirmation).

Conclusion: Our findings suggest a major role of IFN- $\gamma$ and inflammatory monocytes through IL-1 in the pathogenesis of MIS-C, which can be of relevance for the therapeutic management of affected patients. Funded by "Kids Corona" project by Hospital Sant Joan de Déu.

\section{Consent}

I have obtained written consent

Disclosure of Interest

None declared

\section{LB003}

Covid-19 Pandemic - Related Chilblains: Clinical and Immunological Characterization of an Italian Cohort

S. Signa 1,2, F. Manunza ${ }^{3}$, C. Pastorino ${ }^{3}$, P. Bocca' ${ }^{1}$, A. Corcione', F. Penco', A. Bertoni ${ }^{1}$, G. Viglizzo ${ }^{3}$, S. Rosina ${ }^{4}$, P. Giordano ${ }^{2}$, C. Occella ${ }^{3}$, M. Acquila ${ }^{5}$, C. Medici ${ }^{5}$, E. Castagnola², M. Gattorno', S. Volpi

${ }^{1}$ UOSD Centro Malattie Autoinfiammatorie e Immunodeficienze; ${ }^{2}$ UOC Malattie Infettive; ${ }^{3} \cup O$ Dermatologia; ${ }^{4} \cup O C$ Clinica Pediatrica e Reumatologia; ${ }^{5}$ UOC Laboratori Analisi, IRCCS Istituto Giannina Gaslini, Genoa, Italy

Correspondence: $\mathrm{S}$. Signa

Pediatric Rheumatology 2020, 18(Suppl 3):LB003
Introduction: During COVID-19 pandemic, acute acral chilblainlike lesions (ACBLL), reminiscent of lupus pernio, were initially observed among patients with highly suspected (but mostly unconfirmed) infection with SARS-CoV-2.The aetiology of this phenomenon has not been elucidated yet and pathogenetic mechanism remains unknown. Several studies have investigated cytokine and chemokine profile in patients with COVID-19 but a characterization of ACBLL patients is lacking.

Objectives: We aimed to describe the clinical, laboratory and immunological features of children presenting with ACBLL referred to our Institute during the COVID-19 pandemic spread. Methods: We prospectively collected data of children referred to our Institute from April 1st to June 30th. We investigate the presence of SARS-CoV2 infection through RT-PCR from nasopharingeal swabs and two different serologic kit. Nine patients accepted to be studied prospectively. All patients underwent a laboratory work-up including coagulation, viral serology and autoantibodies panel. Finally, we analysed peripheral blood IFN signature and a panel of inflammatory biomarkers in serum/plasma by a flow cytometry bead array (CXCL10, CXCL9, IL-6, IL-1 $\beta, T N F a)$. Skin biopsy was not performed because of our Institute restriction measures adopted during the pandemic.

Results: We examined 31 children ( $F$ : 20; median age 12 y), at a median delay of 26 days after symptoms onset (2-73 days). Twelve patients (39\%) presented non-specific systemic symptoms preceding ACBLL onset. Four patients (13\%) reported a possible contagion from a close contact. All patients presented stereotypical features resembling classical chilblains with acral erythematous-edematous violaceous plaques and nodules localized on the toes $(n=23,74 \%)$, the fingers $(n=4,13 \%)$ or on both sites $(n=4,13 \%)$. SARS-CoV-2 RNA detection resulted negative except for 2 patients. Repeated SARS-CoV-2 specific $\mathrm{IgG} / \mathrm{lgA}$ tests were negative for all patients except for the two cases with positive swabs who showed IgG positivity; one of them was also positive for $\lg A$. Neither common virus serology nor coagulation studies revealed significative results. Two patients presented positive ANA and anti $\beta 2$ glycoprotein,respectively. A positive IFN signature was detected only in 1/ 27 patients (4\%).The cytokine array showed high levels of IP10 $(n=7$, range $41-534 \mathrm{pg} / \mathrm{ml}, \mathrm{n} . v \cdot 0.0-0.2 \mathrm{pg} / \mathrm{ml})$ and a mild increase of IL-6 $(\mathrm{n}=$ 5 , range $3.2-6.5 \mathrm{pg} / \mathrm{ml}$, n.v. $0.5-2.2 \mathrm{pg} / \mathrm{ml}$ ), without alterations of CXCL9, IL-1 $\beta$ and TNFa.Nine patients agreed to further follow-up after a median of 32 days (22-61 days) from the first visit. No seroconversion was observed among them. In addition, 4/6 tested patients (66\%) showed a reduction of IL-6 levels at follow-up.

Conclusion: Albeit the role of SARS-CoV-2 in the development of ACBLL remains to be elucidated, our preliminary results showed a significant increase in serum IP10 levels, not associated with a peripheral blood IFN signature, which is instead a characteristic of pernio-related chilblains. IP10 secretion is induced by type I and type II IFN and lack of type I IFN signature in peripheral blood opens at least two scenarios: a predominant role for type II IFN or a more local type I IFN activation, possibly triggered by the virus itself and not involving circulating blood cells, thus explaining lack of systemic symptoms in most of these patients.

\section{Disclosure of Interest \\ None declared}

\section{LB004}

The Effectiveness of Post-Isometric Muscle Relaxation in Children and Adolescents with Ankylosing Spondylitis

O. A. Tikhoplav', E. Guryanova²

${ }^{1}$ Chuvash State University, ${ }^{2}$ Republican Clinical Hospital, Cheboksary,

Russian Federation

Correspondence: $O$. A. Tikhoplav

Pediatric Rheumatology 2020, 18(Suppl 3):LB004 
Introduction: Ankylosing spondylitis (AS) is a chronic, gradually progressive inflammatory disease of the spine, which in a number of patients can occur simultaneously with lesions of entheses and peripheral joints.

Objectives: The aim of the study was to compare the effectiveness of treatment of juvenile forms of AS, with the use of postisometric relaxation techniques in the treatment program, and without it.

The onset of the disease often occurs in childhood (juvenile AS). At the same time, in childhood, the manifestations of peripheral arthritis and enthesitis significantly prevail in patients over the symptoms of axial lesion, and in adolescence - the pathology of the hip joints (coxitis). Subsequently, the clinical picture of the disease becomes more typical for AS.

The aim of the study was to compare the effectiveness of treatment of juvenile forms of AS, with the use of post-isometric relaxation techniques in the treatment program, and without it.The aim of the study was to compare the effectiveness of treatment of juvenile forms of AS, with the use of postisometric relaxation techniques in the treatment program, and without it.

Methods: Methods. A prospective clinical study was conducted with 98 children with a confirmed diagnosis of ankylosing spondylitis aged 10 to 14 years (68 girls, 30 boys). All patients had a period from the moment of diagnosis from 6 months to one and a half years. The exclusion criteria for patients were the presence of other diseases, joints and / or spine, which aggravated the course of the underlying disease. All children were randomly divided into two groups: children (68) who received a combination treatment including massage, acupuncture and exercise and post-isometric muscle relaxation techniques and children (30) of the 2 nd group (G2) who received the program, with the exception of post-isometric relaxation.

All children received treatment 5 times a week (from Monday to Friday, except Saturday and Sunday), the total duration of the rehabilitation course was 14 days. The therapeutic program included the following non-drug methods of exposure: for $\mathrm{G} 1,30$ minutes - an acupuncture session, 20 minutes - a general massage session and at least 45 minutes of exercises in a gymnastics room with a physiotherapy instructor, as well as sessions of post-isometric muscle relaxation for the chest, back, upper and lower limbs. The visual analogue scale (VAS) was used as a method of assessing effectiveness, which was assessed at the beginning of the study, at the fifth, ninth and last clinic visits. Also, the BASDAl index was used.

Results: Results. 97 patients (99\%) completed the protocol: 68 $(100 \%)$ in G1 and 29 (97\%) in G2. The average VAS score in G1 at the beginning of the study was $5.8 \pm 0.6$, and after the tenth session it dropped to $2.1 \pm 0.3$. A significant decrease in pain was also recorded in group G2 (from $5.8 \pm 0.6$ at the beginning of the study to $3.5 \pm 0.2$ after treatment), a statistically significant difference $(p<0.05)$ between different groups. To assess the period of time over which the treatment effect persists, all patients were asked to send a subjective VAS score to the treating physician after completing treatment on a monthly basis for 12 months. It was found that the inclusion of post-isometric muscle relaxation in the rehabilitation program allows the effects of pain reduction to be prolonged. Patients in the G1 group had lower VAS scores compared to patients in the G2 group by 4.3 months longer.

Conclusion: Conclusion. The results obtained indicate a high efficiency of the use of post-isometric muscle relaxation in the complex treatment of patients with established ankylosing spondylitis.

\section{Disclosure of Interest}

None declared

\section{LB005}

Canakinumab in Systemic Juvenile Idiopathic Arthritis: Clinical Inactive Disease Rate and Safety in Italian Patients

A. De Matteis', C. Bracaglia', D. Pires Marafon', M. Alessio², G. Filocamo³, F. Minoia ${ }^{3}$, J. Tibaldi ${ }^{4}$, A. Marino ${ }^{5}$, G. Simonini ${ }^{5}$, F. La Torre ${ }^{6}$, M. C. Maggio ${ }^{7}$, C. Alizzi $^{8}$, F. Licciardi ${ }^{9}$, D. Montin ${ }^{9}$, G. Martini ${ }^{10}$, A. Civino ${ }^{11}$, G. Romina ${ }^{12}$, A. N. Olivieri $^{13}$, E. Cortis ${ }^{14}$, G. Conti ${ }^{15}$, F. Orlando ${ }^{16}$, R. Naddei ${ }^{2}$, S. Martino ${ }^{9}$, A. L. Piscitelli ${ }^{1}$, R. Cimaz ${ }^{17}$, A. Ravelli ${ }^{4}$, F. De Benedetti' ${ }^{1}$, M. Pardeo'

${ }^{1}$ Division of Rheumatology, IRCCS Ospedale Pediatrico Bambino Gesù, Roma; ${ }^{2}$ Department of Translational Medicine, University Naples Federico II, Napoli; ${ }^{3}$ Intermediate Pediatric Care Unit, Fondazione IRCCS Ca' Grande Ospedale Maggiore Policlinico, Milano; ${ }^{4}$ Division of Rheumatology, University of Genova and Giannina Gaslini Institute, Genova; ${ }^{5}$ Department of Neurosciences, Psychology, Drug Research and Child Health, Rheumatology Unit, Meyer Children's Hospital, University of Florence, Firenze; ${ }^{6}$ Department of Pediatrics, division of Rheumatology, Ospedale "Giovanni XXIII", University of Bari, Bari; ${ }^{7}$ University Department Pro.Sa.M.I. "G. D'Alessandro"; ${ }^{8}$ University Department Pro.Sa.M.I "G. D'Alessandro", University of Palermo, Palermo; ${ }^{9}$ Department of Pediatrics and Infectious Diseases, School of Medicine, University of Turin, Regina Margherita Children's Hospital, Torino; ${ }^{10}$ Department of Woman and Child Health, University of Padua, Padova; ${ }^{11}$ Department of Pediatrics,

division of Rheumatology and Immunology, Ospedale "Vito Fazzi", Lecce; ${ }^{12}$ Unit of Pediatric Nephrology and Rheumatology, University of Messina, Messina; ${ }^{13}$ Department of Woman, Child, and General and Specialistic Surgery, Second University of Studies of Naples, Napoli; ${ }^{14}$ Department of Pediatrics, Sant' Eugenio Hospital, Roma; ${ }^{15}$ Pediatric Nephrology and Rheumatology Unit, AOU Policlinico "G. Martino", Messina; ${ }^{16}$ Unit of

Pediatrics 2, Santobono-Pausilipon Children's Hospital, Napoli; ${ }^{17}$ Research Center for Adult and Pediatric Rheumatic Diseases, University of Milan,

Milano, Italy

Correspondence: A. De Matteis

Pediatric Rheumatology 2020, 18(Suppl 3):LB005

Introduction: Systemic juvenile idiopathic arthritis (sJIA) accounts for $10-20 \%$ of all patients with JIA. The demonstration of a key role of IL- 1 and IL- 6 in the pathogenesis of the disease, led to consider SJIA an autoinflammatory disease: this explain the successfully use of IL-1 and IL- 6 inhibitors. While the efficacy and safety of anakinra in SJIA is widely documented, there are no reports on large series of patients treated with canakinumab outside of the setting of clinical trials.

Objectives: The aim of this study was to evaluate clinical response rate and disease course of canakinumab in Italian cohort of patients with sJIA.

Methods: This is a retrospective multicenter study. Demographic features, previous medical history and therapies was evaluated for each patients. Clinical features, laboratory parameters and adverse events were collected at baseline and after 6 months from starting canakinumab. Clinically inactive disease (CID) was defined according to Wallace criteria.

Results: We enrolled $82(50 \mathrm{~F})$ patients with sJIA treated with canakinumab from 2006 to 2020; 75 of them reached a follow up of 6 months. At baseline 49 patients (59.8\%), of which 36 in active disease $(A D)$ and 13 in $C I D$, were previously treated with anakinra, while 33 patients in $A D(40.2 \%)$ were naïve. At 6 months of follow-up 51/75 patients (68\%) met criteria of CID off-glucocorticoids, including all 13 patients in CID at baseline, 19 patients in $A D$ previously treated with anakinra and 19 patients naïve. Twenty-four patients (32\%) maintained AD. To evaluated if the response to canakinumab might be related to the baseline features we excluded 13 patients in CID at baseline; we divided the 62 patients in responders $(38 / 62,61.3 \%)$ and non-responders $(24 / 62,38.7 \%)$. There were no significant differences between the two groups regarding demographic, clinical and laboratory parameters, except for a higher number of active joints $(p=0.021)$ and for a greater use of disease-modifying antirheumatic drugs (DMARDs) $(p<0.0001)$ in non-responders patients (Table). No major adverse events nor cases of macrophage activated syndrome were recorded. 
Conclusion: Canakinumab was able to induce CID in patients in $A D$ at baseline (both in naïve patients and in patients previous treated with anakinra) and to maintain clinical remission achieved with anakinra. The percentage of clinical response is in keeping with what reported in literature, even if we did not found predictive factors of response.

Trial registration identifying number:

The study was performed after approval by the ethics committee of the "Ospedale Pediatrico

Bambino Gesù" with the ethics approval number 1683 OPBG 2018.

The study was performed after approval by the ethics committee of the "Ospedale Pediatrico Bambino Gesù" with the ethics approval number 1683 OPBG 2018.

Consent

I have obtained written consent

\section{Disclosure of Interest}

None declared

\section{LB006}

Risk Factors of Persistently Active Disease among Filipino Children with Systemic Juvenile Idiopathic Arthritis: 10-Year Study in a

Tertiary Hospital

I. J. E. Alberca, M. T. M. Collante, C. B. Bernal

Pediatrics, UNIVERSITY OF SANTO TOMAS HOSPITAL, Manila, Philippines Correspondence: $\perp$. J. E. Alberca

Pediatric Rheumatology 2020, 18(Suppl 3):LB006

Introduction: Systemic Juvenile Idiopathic Arthritis (SJIA) is one of the most common subtypes of arthritis among children in Southeast Asia with higher progression of disease activity. Unsuccessful control of the disease may lead to long-term disability resulting to functional limitations that would affect the productivity of the individual.

Objectives: The study determined the risk factors for persistently active disease among Filipino children aged 2 weeks to 18 years diagnosed with SJIA seen in the Section of Pediatric Rheumatology of University of Santo Tomas Hospital (USTH) from June 2009 to June 2019.

Methods: A retrospective cohort study was done involving chart review of both clinical division and private division patients. The following parameters were determined: sex, age at diagnosis, time elapsed from symptom onset to diagnosis, joint involvement, inflammatory markers, and extra-articular manifestation. Statistical analysis included frequencies, percentages, and logistic regression for the risk factors of interest.

Results: One hundred twenty-seven patients with SJIA who were appropriately treated for at least 3 years were included. Among which, $88(69 \%)$ developed a persistently active disease. Among them, $36(41 \%)$ were diagnosed at 1-5 years old. Many were diagnosed $(n=54,61 \%)$ after 5 weeks. The most commonly affected joints were the wrists, knees, and ankles. Most common contracture noted involved the cervical joint. Only 33 (26\%) patients received biologic agents. Risk factors identified for the development of persistent disease activity were low hemoglobin levels at the time of diagnosis and after 1 month of treatment, elevated platelet count after a month, substantial joint count after 3 months, and increased ESR after 6 months.

Conclusion: The change or improvement of the joint count and in hemoglobin, platelet count, and ESR levels after appropriate treatment may determine risk for persistently active disease in Filipino children with SJIA.

Consent

I have obtained written consent

\section{Disclosure of Interest}

None declared

Publisher's Note

Springer Nature remains neutral with regard to jurisdictional claims in published maps and institutional affiliations.
Ready to submit your research? Choose BMC and benefit from:

- fast, convenient online submission

- thorough peer review by experienced researchers in your field

- rapid publication on acceptance

- support for research data, including large and complex data types

- gold Open Access which fosters wider collaboration and increased citations

- maximum visibility for your research: over $100 \mathrm{M}$ website views per year

At $\mathrm{BMC}$, research is always in progress.

Learn more biomedcentral.com/submissions 